\title{
Factors Contributing to Fentanyl Pharmacokinetic Variability Among Diagnostically Diverse Critically III Children
}

\author{
Fanuel T. Hagos ${ }^{1}$. Christopher M. Horvat ${ }^{2,3}$ (1) - Alicia K. Au ${ }^{2,3} \cdot$ Yvette P. Conley ${ }^{4,5}$ - Lingjue $\mathrm{Li}^{3,6}$. \\ Samuel M. Poloyac ${ }^{3,6} \cdot$ Patrick M. Kochanek ${ }^{2,3} \cdot$ Robert S. B. Clark $^{2,3} \cdot$ Philip E. Empey Em, $^{3,6}$
}

Published online: 6 June 2019

(c) Springer Nature Switzerland AG 2019

\begin{abstract}
Objective The objective of this study was to characterize the population pharmacokinetics of fentanyl and identify factors that contribute to exposure variability in critically ill pediatric patients.

Methods We conducted a single-center, retrospective cohort study using electronic record data and remnant blood samples in the setting of a mixed medical/surgical intensive care unit (ICU) at a quaternary children's hospital. Children with a predicted ICU length of stay of at least 3 days and presence of an indwelling central venous or arterial line were included. Serum fentanyl measurements were performed for 278 unique remnant samples from 66 patients. Both one- and two-compartment models were evaluated to describe fentanyl disposition. Covariates were introduced into the model in a forward/backward, stepwise approach and included age, sex, race, weight, cytochrome P450 (CYP) 3A5 genotype, and the presence of CYP3A4 or CYP3A5 inducers or inhibitors. Simulations were performed using the successful model to depict the influence of inducers on fentanyl concentrations.

Results A two-compartment base model best described the data. There was good agreement between observed and predicted concentrations in the final model. The typical fentanyl clearance for $70 \mathrm{~kg}$ (reference weight) and $20.1 \mathrm{~kg}$ (median weight) patients were 34.6 and $13.6 \mathrm{~L} / \mathrm{h}$, respectively. The magnitude of the unexplained random inter-individual variability was high for both clearance $(60.7 \%)$ and apparent volume of the central compartment $\left(V_{1}\right)(107.2 \%)$. Coadministration of the known CYP3A4/5 inducers fosphenytoin and/or phenobarbital was associated with significantly increased fentanyl clearance. Simulations demonstrate that the effect of inducer administration was most pronounced following discontinuation of a fentanyl infusion.

Conclusions In this study we show the feasibility and utility of using electronic record data and remnant blood samples to successfully construct population pharmacokinetic models for a heterogeneous cohort of critically ill children. A clinically relevant effect of concomitant CYP3A4/5 inducers was identified. Scaling this population pharmacokinetic approach is necessary to craft precision approaches to fentanyl administration for critically ill children.
\end{abstract}

Fanuel T. Hagos and Christopher M. Horvat contributed equally to this article.

Electronic supplementary material The online version of this article (https://doi.org/10.1007/s40262-019-00773-1) contains supplementary material, which is available to authorized users.

Christopher M. Horvat

Christopher.horvat@chp.edu

Extended author information available on the last page of the article

\section{Key Points}

Population pharmacokinetic modeling is a useful approach to better understand drug kinetics among critically ill children.

Medications that induce expression of cytochrome $\mathrm{P} 450$ $3 \mathrm{~A} 4$ and $3 \mathrm{~A} 5$ significantly influence fentnayl clearance in critically ill children. 


\section{Introduction}

Strategies for selecting a specific sedative-analgesic drug and dose in the pediatric intensive care unit (PICU) are largely rooted in empiricism, guided by institutional standards, individual experience, and heuristic estimates of what a patient will require for adequate comfort and safety. A large study comparing a sedation protocol versus usual sedation in children with respiratory failure did not demonstrate any difference in the primary outcome, duration of mechanical ventilation [1]. The relationship between wakefulness, pain, agitation, iatrogenic drug dependence, and adequate sedation-analgesia in children is variable, influenced largely by pharmacokinetics and pharmacodynamics. Unfortunately, these remain poorly characterized for most commonly used drugs in the PICU.

Fentanyl is a highly lipid-soluble opioid frequently favored in the PICU for continuous sedation-analgesia owing to its observed relatively neutral hemodynamic effects [2]. Characterizing fentanyl pharmacokinetics and factors associated with variable dose requirements among critically ill children is challenging due to the dense sampling strategy required by traditional pharmacokinetic studies being obviated by caregiver stress and concern for iatrogenic anemia in the setting of critical illness. Several small studies have reported a range of pharmacokinetic parameters in children admitted to the PICU, though the limited cohort sizes do not sufficiently account for the patient and clinical heterogeneity inherent in this population [3]. The largest fentanyl pharmacokinetic study in children used population-level modeling methods to characterize pharmacokinetic parameters from sparse samples; however, this study was restricted to a relatively homogenous cardiac intensive care unit (ICU) population with a median age less than 1 year old [4].

Conventionally, fentanyl is dosed in micrograms per kilogram $(\mu \mathrm{g} / \mathrm{kg})$ in pediatrics, with standard bolus doses ranging widely from approximately $1-10 \mu \mathrm{g} / \mathrm{kg}$ and infusions commonly initiated at $0.5-2 \mu \mathrm{g} / \mathrm{kg} / \mathrm{h}$. The therapeutic window of fentanyl has been reported to be relatively narrow, with serum concentrations associated with mild analgesia reported to be $0.6 \mathrm{ng} / \mathrm{mL}$ and substantial analgesia reported at concentrations ranging from 1.2 to $3 \mathrm{ng} / \mathrm{mL}$ among adults $[5,6]$. The fentanyl dose response among critically ill children is reported to be highly variable and standard approaches are to administer doses until a desired clinical effect is achieved [7, 8]. At least part of this variability in response is related to fentanyl being metabolized predominantly by the hepatic cytochrome P450 (CYP) enzyme CYP3A4, the activity of which demonstrates high inter-individual variability and evolves with age $[3,9]$. The objective of this study was to construct a population pharmacokinetic model for fentanyl and to identify demographic and clinical covariates that affect the pharmacokinetic parameters of fentanyl in a clinically diverse cohort of critically ill children. Modeling fentanyl pharmacokinetics among children in the PICU is a necessary step towards more individualized dosing strategies, with the ultimate goal of reducing episodes of inadequate sedation-analgesia and optimizing the safety of these patients.

\section{Methods}

\subsection{Study Design and Population}

A retrospective cohort study was undertaken involving patients admitted to the PICU at our quaternary children's hospital. Approval was granted by the University of Pittsburgh Institutional Review Board (Pittsburgh, PA, USA). Fentanyl is administered intravenously as a continuous infusion and/or as an intermittent bolus. All aspects of the administration of fentanyl, including doses of both the infusion and boluses, were determined by the clinical staff. Serum samples and DNA were previously banked as part of a previous cohort study of randomly enrolled PICU patients; inclusion criteria included predicted PICU length of stay $\geq 3$ days, presence of an indwelling central venous or arterial line for blood sampling, and obtainment of informed consent from the parent or legal guardian, with inclusion otherwise unrestricted. Serum samples and blood pellets were stored at $-80^{\circ} \mathrm{C}$. Each serum sample used for analyses contained at least $100 \mu \mathrm{L}$. Samples were collected at unspecified times that were convenient for nurses responsible for collection, without an a priori plan to use the samples for pharmacokinetic analyses and independent of drug administration. DNA was extracted using the QIAamp ${ }^{\circledR}$ DNA extraction kit (Qiagen Inc., Valencia, CA, USA).

Demographics and clinical characteristics were extracted from an electronic health record (EHR) data warehouse using the business intelligence platform $\mathrm{SAP}^{\circledR}$ BusinessObjects $^{\mathrm{TM}}$ (SAP, Waldorf, Germany). The primary admission diagnosis for each patient was categorized as neurologic, respiratory, sepsis, gastrointestinal disease including hepatic and bowel transplant recipients, or other. The discrete fentanyl dose amount and administration timing were determined from the patient medication administration record. Biomarker levels of interest were extracted from the data warehouse and included alanine and aspartate aminotransferase, serum albumin, and creatinine. All administered medications were reviewed and, in addition to fentanyl, medications of interest selected for inclusion in the models were previously reported CYP450 enzyme inducers fosphenytoin, phenytoin, and phenobarbital and the inhibitor fluconazole, 
provided the inhibitor/inducer had been administered for at least $48 \mathrm{~h}$ prior to administration of fentanyl.

\subsection{Drug Concentration Assessment and Population Pharmacokinetic Analysis}

Serum fentanyl concentrations were measured using a sensitive and specific validated liquid chromatography-tandem mass spectrometry (LC-MS/MS) method that had been previously described [10]. Measured levels above the limit of quantification were excluded from the study. Levels within $15 \%$ of the lower limit of quantification were included in the primary analysis. A separate sensitivity analysis was performed excluding levels below the lower limit of quantification. NONMEM $^{\circledR}$ version 7.3 software (ICON Development Solutions, Ellicott City, MD, USA) was used to perform the non-linear mixed effects analysis. The software tools and packages Pirana $^{\circledR}$, Perl-speaks-NONMEM (PsN), Xpose4, Xpose (Uppsala University, Pharmacometrics Research Group, Uppsala, Sweden), Excel ${ }^{\circledR}$ (Microsoft Corp., Redmond, WA, USA), R version 3.3.3 (R Foundation for Statistical Computing, Vienna, Austria), and OpenRefine (version 2.7) were used to facilitate dataset preparation, analysis, bootstrap analysis, visual predictive check (VPC) analysis, and preparation of graphical outputs [3, 11-13]. Parameters were estimated using the first-order conditional estimation with interaction (FOCE-I) method. An exponential model, $P_{i j}=\mathrm{TV}\left(P_{j}\right) \exp \left(\eta_{i j}\right)$, was used to describe the variability between individual parameters where $\eta_{i j}$ is the random effect of the $i$ th individual and the $j$ th parameter, which is assumed to be normally distributed with a mean of 0 and variance of $\omega^{2}$.

The residual variability was modelled using the additive model after log-transformation of both sides. Although previous studies have reported that fentanyl follows a twocompartment disposition model, a one-compartment model was also explored $[4,14]$. The effect of patient weight on clearance (CL) and volume of distribution terms were incorporated in the base model by allometric scaling using $P_{i}=P_{\mathrm{STD}} \times\left(W_{i} / W_{\mathrm{STD}}\right)^{\mathrm{PWR}}$, where $P_{i}$ and $W_{i}$ are the pharmacokinetic parameters and weight of the $i$ th individual, respectively. $W_{\mathrm{STD}}$ is the reference weight, set to $70 \mathrm{~kg}$ for these analyses, and $P_{\mathrm{STD}}$ is the pharmacokinetic parameter of the individual of the reference weight. The PWR exponent was fixed at 0.75 for CL terms and 1 for volume terms.

Age, race, sex, CYP3A5 rs28365094 genotype, and concomitant administration of CYP3A4/5 inducers or inhibitors were investigated for their effect on CL. On the basis of a previously reported association with a CYP $3 A 5$ single nucleotide polymorphism at rs 28365094 , covariate evaluation included attempting to model dichotomized genotype, TT versus CT + CC, at this locus [15]. Race was dichotomized into white and non-white and the use of a CYP3A4/5 inducer or inhibitor was dichotomized into present or absent. Power models were used to describe the relationship between covariates and the typical value of CL. A sigmoidal maturation function $\left[\mathrm{CL}=\left(\mathrm{CL}_{\text {typical-value }} \times \mathrm{WT} / 70\right.\right.$ )$^{0.75} \times\left(\mathrm{Age}^{\mathrm{h}} / \mathrm{Age}_{50}{ }^{\mathrm{h}}+\mathrm{Age}^{\mathrm{h}}\right)$, where WT is weight] was also attempted to describe the effect of age using postnatal age since gestational age was not available. All continuous variables were normalized by the median value of the covariate in the cohort. Covariates were added to a model by multiplicative relationship.

Covariate selection was performed using a stepwise approach. The process began with forward addition, in which covariates are added one at a time and considered significant if the covariate contributes to greater than 3.841 reduction in the objective function $(\alpha=0.05,1$ degree of freedom) when added to the previous model. The covariate that improved the model the most is added first and is kept in the model if it fulfills the significance test. The resulting model serves as the base mode for the addition of the next covariate step. These steps were repeated until there were no significant remaining covariates. A backward elimination step was then performed in which a covariate was considered significant if it contributed to greater than a 6.635 magnitude increase in objective function $(\alpha=0.01,1$ degree of freedom) when removed from the previous model. These steps were repeated until there were no non-significant covariates remaining. Assessments of the fit of the pharmacokinetic models were performed by visual inspection of diagnostic plots, successful minimization, VPCs, and changes in objective function values. The stability of the final model and the precision of the parameter estimates were internally validated by bootstrapping in PsN. One thousand replicates were generated by random sampling with replacement from the original dataset. The non-parametric statistics [i.e., median and $95 \%$ confidence interval (CI)] of the parameter estimates generated by bootstrap were compared to the point estimates obtained from the model.

To demonstrate the effect of concomitant administration of CYP3A4/5 inducers, concentration-time profiles of fentanyl were simulated for 200 patients with a median weight of $22 \mathrm{~kg}$ in the presence and absence of inducer administration in the preceding $48 \mathrm{~h}$. For this simulation it was assumed that induction of fentanyl metabolism was in effect at the initiation of fentanyl administration (i.e., subjects who received a CYP3A4/5 inducer for at least $48 \mathrm{~h}$ prior to the start of a fentanyl infusion). It was also assumed that administration of fentanyl began with a bolus dose $(1 \mu \mathrm{g} / \mathrm{kg})$ followed by initiation of an infusion $(1 \mu \mathrm{g} / \mathrm{kg} / \mathrm{h}) 30 \mathrm{~min}$ afterwards. Two subsequent boluses $(1 \mu \mathrm{g} / \mathrm{kg})$ were also simulated, at 2 and $9 \mathrm{~h}$ after initiation of the infusion, and the infusion rate $(1 \mu \mathrm{g} / \mathrm{kg} / \mathrm{h})$ remained constant and was continued for $48 \mathrm{~h}$ after receipt of the second bolus to simulate the steady state. Finally, the trajectories of the fentanyl concentrations 
were examined for an additional $24 \mathrm{~h}$ following cessation of the infusion to observe the distribution and elimination phases in the presence and absence of an inducer. Concentration-time plots of patients in the presence or absence of CYP3A4/5 inducers were created for visual comparison of the effect of inducers.

\section{Results}

A total of 278 samples from 66 patients were used for pharmacokinetic analysis. Baseline demographic and clinical characteristics of the study participants are summarized in Table 1. In 60 of the 66 subjects, samples were collected before, during, and after continuous infusion. In six subjects, all samples were collected only when the subjects had received one or more boluses. One sample with levels above the upper limit of quantification of the assay was excluded from the analysis. Fourteen of the 278 samples were within $15 \%$ of the assay's lower limit of quantification and were included in the analysis.

A two-compartment model with linear elimination, and allometrically scaled weight effects on CL, intercompartmental clearance $(Q)$, apparent volume of the central compartment $\left(V_{1}\right)$, and apparent volume of the peripheral compartment $\left(V_{2}\right)$ was selected as the base model. The final parameter estimates of the model are shown in Table 2. A sensitivity analysis excluding samples below the lower limit of quantification did not result in substantially different estimated parameters (Electronic Supplementary Material Table 1). Retaining the samples resulted in improved model minimization and covariance completion. The typical population pharmacokinetic parameters [estimates (bootstrapped 95\% CI)] relative to the reference patient $(70 \mathrm{~kg}$ and no concomitant administration of CYP3A4/5 inducer or inhibitor) are reported. The typical CL of fentanyl for a $70 \mathrm{~kg}$ subject

Table 1 Cohort characteristics

\begin{tabular}{|c|c|}
\hline Demographics & Cohort $(n=66)$ \\
\hline Weight $(\mathrm{kg})$ [median (range)] & $20.1(4.1-117.4)$ \\
\hline Age (months) [median (range)] & $78(1-204)$ \\
\hline Female $[n(\%)]$ & $34(52)$ \\
\hline \multicolumn{2}{|l|}{$\operatorname{Race}[n(\%)]$} \\
\hline White & $57(86)$ \\
\hline Black & $7(11)$ \\
\hline Other/unknown & $2(3)$ \\
\hline \multicolumn{2}{|l|}{ Primary diagnosis category $[n(\%)]$} \\
\hline Neurologic & $19(29)$ \\
\hline Respiratory & $16(24)$ \\
\hline Sepsis & $6(9)$ \\
\hline Gastrointestinal/transplant & $6(9)$ \\
\hline Other & $19(29)$ \\
\hline Mechanical ventilation $[n(\%)]$ & $61(92)$ \\
\hline Neuromuscular blockade $[n(\%)]$ & $28(42)$ \\
\hline Vasoactive medication infusion $[n(\%)]$ & $25(38)$ \\
\hline PICU length of stay (days) [median (range)] & $8.4(1.1-129)$ \\
\hline Hospital length of stay (days) [median (range)] & $15.5(2.4-129.4)$ \\
\hline Number of samples per patient [median (range)] & $4(1-7)$ \\
\hline Duration of fentanyl infusion (h) [median (range)] & $90.7(11-254)$ \\
\hline Number of fentanyl boluses per patient [median (range)] & $25(4-77)$ \\
\hline Fentanyl plasma concentration (ng/mL) [median (range)] & $2.29(0.11-84.32)$ \\
\hline \multicolumn{2}{|l|}{ Concomitant CYP3A inducer/inhibitor $[n(\%)]$} \\
\hline Fosphenytoin & $13(20)$ \\
\hline Fosphenytoin + phenobarbital & $2(3)$ \\
\hline Fosphenytoin + pentobarbital & $2(3)$ \\
\hline Phenobarbital & $2(3)$ \\
\hline Fluconazole & $5(8)$ \\
\hline \multicolumn{2}{|l|}{ Genotype $[n(\%)]$} \\
\hline CYP $3 A 5$, rs28365094, CT + CC & $14(21)$ \\
\hline
\end{tabular}

CYP cytochrome $\mathrm{P} 450$, PICU pediatric intensive care unit 
Table 2 Population pharmacokinetic model parameters

\begin{tabular}{lllll}
\hline Pharmacokinetic parameter & & Point estimates & \%RSE & Bootstrap median [95\% CI] \\
\hline Fixed & & & \\
$\mathrm{CL}=\theta_{1} \times(\mathrm{WT} / 70)^{0.75} \times \theta_{9}{ }^{\text {INDU }}$ & $\theta_{1}(\mathrm{~L} / \mathrm{h})$ & 34.6 & 9.2 & $34.7[27.7-40.9]$ \\
$\theta_{9}$ & & 1.54 & 10.1 & $1.54[1.2-1.89]$ \\
$V_{1}=\theta_{2} \times(\mathrm{WT} / 70)^{1}$ & $\theta_{2}(\mathrm{~L})$ & 222 & 18.6 & $215[114-330]$ \\
$Q=\theta_{3} \times(\mathrm{WT} / 70)^{0.75}$ & $\theta_{3}(\mathrm{~L} / \mathrm{h})$ & 8.58 & 18.9 & $9.24[3.72-13.32]$ \\
$V_{2}=\theta_{4} \times(\mathrm{WT} / 70)^{1}$ & $\theta_{4}(\mathrm{~L})$ & 311 & 13.1 & $332[150-472]$ \\
Inter-individual variability & & & & \\
$\omega_{\mathrm{CL}}^{2}(\% \mathrm{CV})$ & & $0.37(60.7)$ & 38.2 & $0.34[0.07-0.67]$ \\
$\left.\omega_{V_{1}}^{2} \% \mathrm{CV}\right)$ & $1.15(107.2)$ & 43.1 & $1.15[0.1-2.2]$ \\
$\mathrm{COV}$ & & $0.63(r=0.85)$ & 41.3 & $0.55[0.04-1.22]$ \\
Residual variability & & & 15.4 & $50.6[43.3-59.7]$ \\
Proportional $\left(\% \mathrm{CV}^{\mathrm{b}}\right)$ & 52 & & \\
\hline
\end{tabular}

$\% C V$ percentage coefficient of variation, $C L$ clearance, INDU cytochrome P450 3A4/5 inducer, $Q$ intercompartmental clearance, $R S E$ relative standard error, $V_{l}$ apparent volume of the central compartment, $V_{2}$ apparent volume of the peripheral compartment, $W T$ weight

${ }^{a}$ Residual variability was additive in $\log$ scale

${ }^{\mathrm{b}} \% \mathrm{CV}$ was calculated as square root of variance $\times 100$ without the presence of a CYP3A4/5 inducer was $34.6 \mathrm{~L} / \mathrm{h}$. For a subject weighing $20.1 \mathrm{~kg}$, which is the median weight of the study population, the typical CL was $13.6 \mathrm{~L} / \mathrm{h}$. No enrolled patient received concomitant CYP3A4/5 inhibitor and inducer medications. Among the covariates tested, only the presence or absence of a concomitantly administrated CYP3A4/5 inducer was found to have a significant effect on $\mathrm{CL}$, increasing the $\mathrm{CL}$ value by mean factor of 1.54 . The assessed genotype did not significantly affect CL. Age and weight were highly correlated (Electronic Supplementary Material Figure S1) and including age did not improve the model. Assessment of the percentage of relative standard errors (RSEs) indicated that $\mathrm{CL}, V_{1}, Q, V_{2}$, effect of inducers, and residual variability were estimated with good precision (RSE values of these parameters were less than 30\%). Inter-individual variability of both $\mathrm{CL}$ and $V_{1}$ values were estimated with moderate precision, with RSE values of $38.2 \%$ and $43.1 \%$, respectively. The magnitude of the unexplained random inter-individual variability [percentage coefficient of variation $(\% \mathrm{CV})$ ] remained high for both $\mathrm{CL}$ and $V_{1}$ at $60.7 \%$ and $107.2 \%$, respectively. For all parameters, the median bootstrapped parameter estimates were comparable to the point estimates from the original datasets, showing the model was robust and described the data well. ETA shrinkage, the shrinkage of inter-subject variability, was $10.3 \%$ for $\mathrm{CL}$ and $50 \%$ for $V_{1}$. Epsilon shrinkage, the shrinkage of the proportional intra-subject variability, was $10.8 \%$.

The goodness-of-fit plots are presented in Fig. 1. The population predicted versus observed concentration (Fig. 1a) and the individual predicted versus observed concentration (Fig. 1b) plots indicate a good agreement between the observed and predicted concentration along with sufficiently symmetric distribution and random scatter of datapoints around the line of unity. Slight biases at the low end of the population plot and high end of the individual plot were observed. This was also observed in the individual weighted residual (IWRES) versus individual predicted concentration plot (Electronic Supplementary Material Figure S2). The conditional weighted residuals plotted across population predicted concentration (Fig. 1c) and over time (Fig. 1d) similarly show symmetrical distribution around the line of zero and that the model described the data well. No trends were observed over time, and a slight bias was observed at the lower value of population predicted concentrations. The normal distribution assumption of ETA1 (inter-individual variability for CL) and ETA2 (inter-individual variability for $V_{1}$ ) were valid (Electronic Supplementary Material Figure S3). The VPCs also indicate that the model adequately describes both the data dynamics and dispersion (Fig. 2). There was generally good agreement between observed and simulated serum concentrations across time with the observed fentanyl plasma concentrations contained within the simulated 95\% CI.

Figure 3 shows the simulated effect of concomitant administration of a CYP3A4/5 inducer on fentanyl concentration for 200 patients. As CL increased in the presence of a concomitant inducer, the plasma concentrations of fentanyl are generally lower, as shown in the concentration-time plot (Fig. 3a). The effect was most pronounced following discontinuation of the fentanyl infusion. Figure $3 b$ shows that the steady-state concentration $\left(C_{\mathrm{ss}}\right)$ was also significantly lower $(p<0.0001)$ in patients receiving inducers, although variability remains high. 


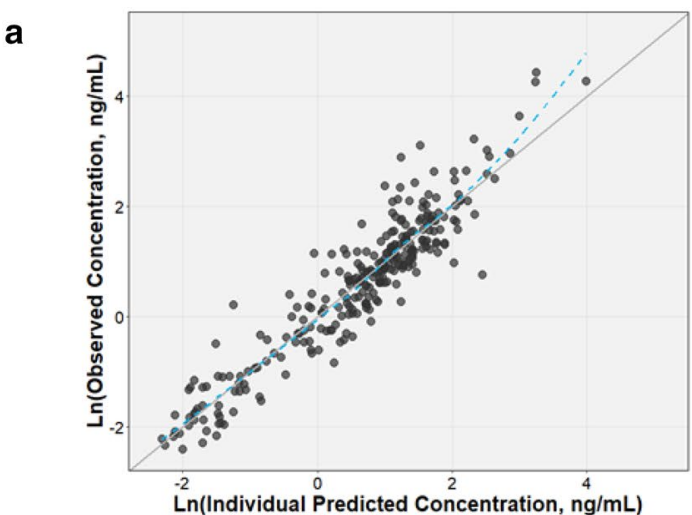

C

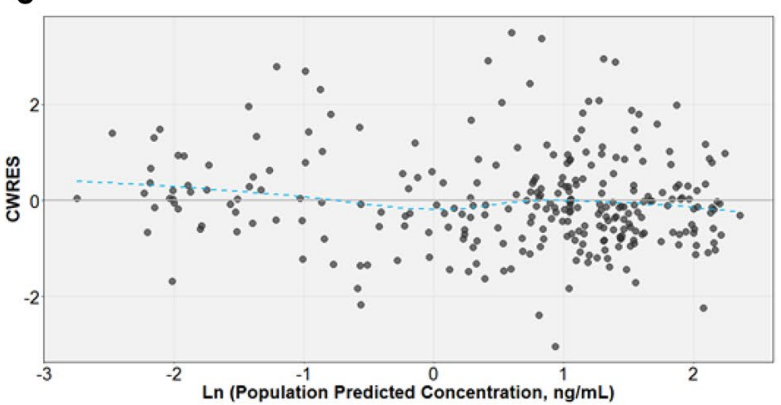

b

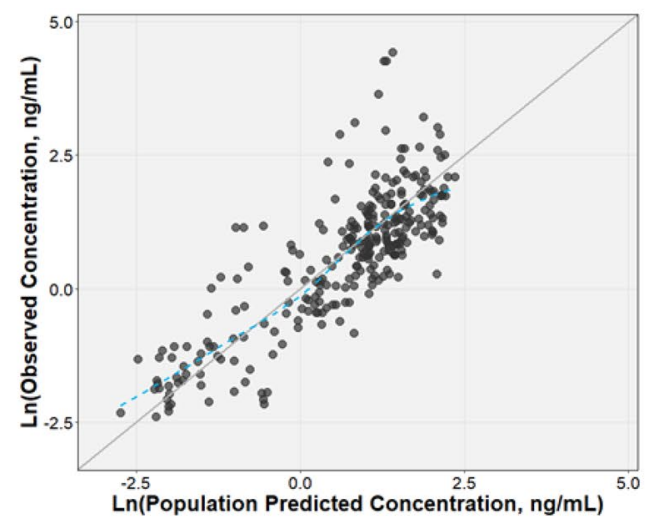

d

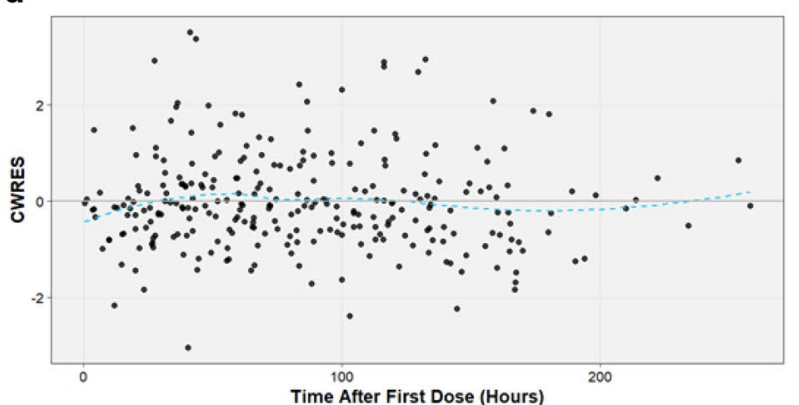

Fig. 1 Population (a) and individual (b) predicted versus observed concentration. Conditional weighted residual versus population predicted concentration (c) and time (d). CWRES population conditional weighted residuals

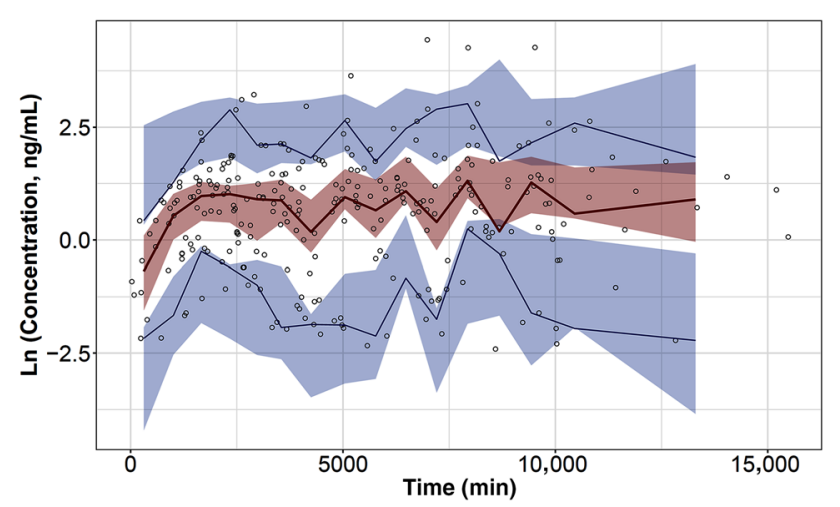

Fig. 2 Standard visual predictive check for the final pharmacokinetic model. Black circles represent the observed data, lines represent the observed median, and upper (95th) and lower (5th) percentile concentrations, and the shaded regions represent the $95 \%$ confidence interval for the simulated median, upper, and lower concentrations

\section{Discussion}

The use of fentanyl is ubiquitous in pediatric critical care, yet factors associated with variable fentanyl dose requirements have not been well-characterized in this heterogeneous patient population, in part due to frequent but impractical sampling requirements for constructing traditional pharmacokinetic models. We leveraged EHR data, sparse remnant blood samples collected at random times, and population pharmacokinetic modeling to successfully describe fentanyl dose-concentration-time in critically ill children. To the best of our knowledge, this study includes the largest cohort of diagnostically diverse, critically ill children enrolled to building a pharmacokinetic model of fentanyl. CL was $13.6 \mathrm{~L} / \mathrm{h}$ for a $20.1 \mathrm{~kg}$ (the median weight for the population in the current study) subject, which is comparable with findings from other critically ill pediatric populations [3, 4]. Concomitant administration of CYP3A4/5 inducers significantly affected the $\mathrm{CL}$ of fentanyl, though the relatively large magnitude of unexplained inter-individual variability in both CL and volume of distribution indicate that other non-modeled factors were influencing dose requirements in this heterogeneous population. The findings of this study demonstrate the feasibility of population pharmacokinetic modeling as a foundational element towards more precise prescribing of fentanyl in the PICU.

A recent systematic review of pharmacokinetic parameters for fentanyl and its derivatives in children identified 14 studies with published CL values ranging from 0.41 to $68.5 \mathrm{~mL} / \mathrm{min} / \mathrm{kg}$ and cohort sizes ranging from one to 130 included patients [3]. Our selection of a two-compartment model was compatible with other studies examining fentanyl pharmacokinetics in children. The largest study used a population pharmacokinetic approach to characterize 
a

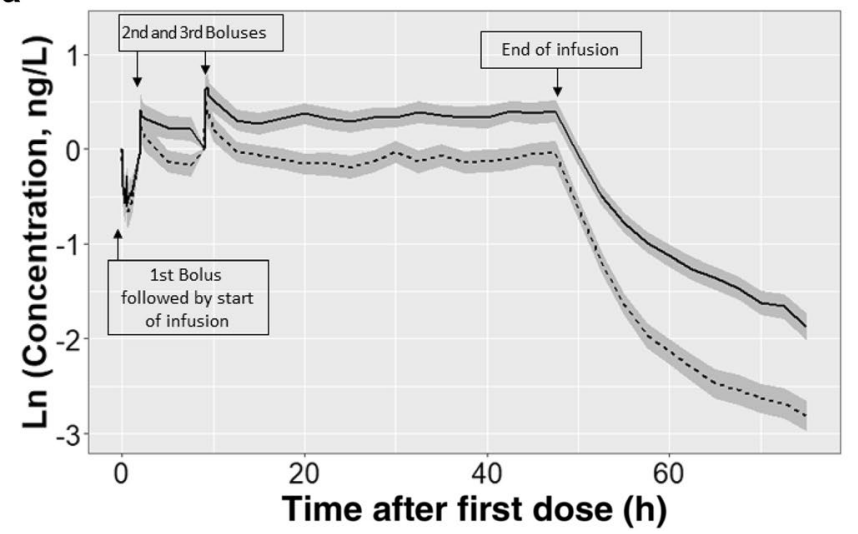

b

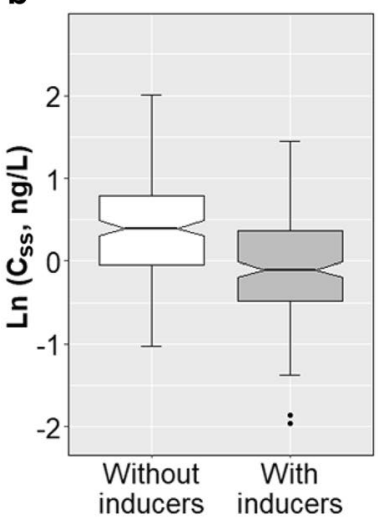

Fig. 3 Simulated concentration-time profile of fentanyl in the presence (dotted line) or absence (solid line) of cytochrome P450 (CYP) $3 \mathrm{~A} 4 / 5$ inducers (a). The lines indicate the mean values while the shaded areas indicate $95 \%$ confidence intervals. Box-plot comparing the distribution of $C_{\mathrm{ss}}$ (steady state concentration) values with

pharmacokinetic parameters for children admitted to a cardiac ICU, had a median age of 5.9 months, and reported a $\mathrm{CL}$ of $39.6 \mathrm{~L} / \mathrm{h}$, which is comparable with the findings of the present work $(34.6 \mathrm{~L} / \mathrm{h})$ [4]. We opted for a two-compartment model of fentanyl, in alignment with other fentanyl pharmacokinetic studies, though some studies have proposed a three-compartment model as more representative of fentanyl distribution $[4,14,16-19]$. A three-compartment model has been proposed to represent initial fentanyl exposure in well-perfused end-organs, with subsequent redistribution to skeletal muscle, followed by slower distribution to adipose tissue. Such a model is supported by preclinical data collected with dense sampling strategies and higher doses than conventionally used in clinical settings, facilitating detection of a third slope of elimination [20]. The sparse sampling strategy of the population pharmacokinetic modeling approach may obscure detection of this third compartment, instead offering the advantage of pragmatic sample collection and larger cohort sizes while still yielding clinically relevant models.

We chose to include weight in the base model given the weight-normalized dosing common in pediatrics. Age and weight are highly correlated and much of the influence of age on fentanyl pharmacokinetics can be accounted for with weight; however, our model does not account for the influence of age exclusive of weight on the pharmacokinetic parameters. The previously observed relationship between fentanyl pharmacokinetics and age has been hypothesized to be related to differences in volume of distribution given the lower concentrations observed in infants versus older patients soon after initiation of an infusion [16]. Mean body fat in a full-term neonate is $12 \%$, increases to approximately $30 \%$ by 1 year of age, and subsequently decreases, though or without coadministration of CYP3A4/5 inducers (b). Patients on inducers at the same time as fentanyl have significantly lower $C_{\mathrm{ss}}$ than those not on inducers. Each of the fentanyl bolus doses were $1 \mu \mathrm{g} /$ $\mathrm{kg}$ while the infusion was administered at $1 \mu \mathrm{g} / \mathrm{kg} / \mathrm{h}$. $C_{s s}$ steady-state concentration

the obesity epidemic in many developed nations has altered the historically observed decline of body mass index (BMI) in older children and adolescents [21-23]. The high lipid solubility of fentanyl is expected to interact significantly with body fat composition and total body water content. A population pharmacokinetic study of fentanyl among critically ill adult patients identified liver disease, congestive heart failure, and weight as factors strongly influencing pharmacokinetics [14]. The median (range) of weight in our cohort was $20.1 \mathrm{~kg}(4.1-117.4 \mathrm{~kg})$, which is representative of patient heterogeneity that is commonplace in the PICU and relatively unique to this arena of medicine. A recent review noted only a weak association between weight and fentanyl CL in preterm and term neonates and acknowledged a lack of data in older children [3]. A relationship between age, adiposity, and fentanyl requirements has some supporting evidence and strong biologic plausibility, indicating the need for larger population pharmacokinetic cohorts with sufficient power to control for body fat, body water, and muscle mass.

Fentanyl is a high-extraction drug with CL largely determined by hepatic blood flow [24]. This property is thought to reduce the likelihood of drug-drug interactions given the expected marginal influence of the major hepatic drug metabolism enzymes CYP3A4 and CYP3A5 [25]. However, the extraction ratio for fentanyl has only been studied in healthy volunteers, making drug CL in the context of critical illness and with multiple coadministered medications uncertain [26]. Fosphenytoin, phenytoin, and phenobarbital are commonly used anticonvulsant medications in pediatric critical care. These drugs are also established inducers of hepatic CYP enzymes. CYP3A4 is involved in the metabolism of approximately $50 \%$ of all marketed medications and 
CYP3A4/5 enzymes play the major role in fentanyl metabolism [3, 9, 27-31]. Xenobiotic response elements exist in regions of $C Y P$ genes and, when bound by a ligand, activate gene transcription [31]. Ligand-bound forms of pregnane $X$ receptor and closely related constitutive androstane receptor each form a heterodimer with retinoid $\mathrm{X}$ receptor, ultimately binding xenobiotic response elements in promoter regions and inducing transcription [31-33]. Both (fos)phenytoin and barbiturates are combined pregnane $\mathrm{X}$ and constitutive androstane receptor activators, inducing CYP3A4/5 [31, 34-37].

Though this inducer effect has been mechanistically well-defined, the clinical impact among intensive care patients has not been studied and the time course of clinical drug-inducer effects is unknown. The effect of drug metabolism enzyme inducers on fentanyl $\mathrm{CL}$ and the time course of these inducer effects has only been evaluated in adults under controlled conditions [38]. Among critically ill children, the effects of patient factors and other coadministered medications are expected to influence the time course of enzyme induction response, though the relationship is not clear. Inducers were included in our model if they had been administered at least $48 \mathrm{~h}$ prior to obtaining a fentanyl concentration, though our study was not designed to parse out the magnitude of effect related to preceding duration of inducer administration. Using simulation, we demonstrated that $C_{\mathrm{ss}}$ values of fentanyl are significantly lower in the presence of CYP3A4/5 inducers. Interestingly, the difference in median concentrations between patients with or without concomitant use of CYP3A4/5 inducers was more pronounced once the infusion was terminated than during steady state (Fig. 3a). After termination of the infusion, the distributionphase half-life $\left(t^{1} / 2 \alpha\right)$ and terminal-phase half-life $\left(t^{1} / 2 \beta\right)$ in the presence of CYP3A4/5 inducers decreased by $29 \%$ and $8 \%$, respectively, as compared to when there were no concomitantly administered CYP3A4/5 inducers. This suggests that the pronounced change in concentrations after termination of infusion was mainly driven by the fact that, in the presence of CYP3A4/5 inducers, fentanyl is being cleared from the central compartment more rapidly with less time to distribute to the peripheral compartment.

The clinical implication of these observations is that patients on CYP3A4/5 inducers may need higher doses and/or infusion rates of fentanyl to obtain the desired drug effect. Also, clinicians interrupting fentanyl infusions during care should consider the faster rate at which fentanyl concentrations decrease in the presence of CYP3A4/5 inducers. Because of the high degree of inter-individual variability, additional study with larger cohorts and more covariates is necessary to establish fentanyl pharmacokinetic models capable of providing pre-emptive guidance on dose adjustment in the setting of inducer initiation. That $C_{\mathrm{ss}}$ values were observed to be lower in the presence of an inducer may be a clinically favorable effect in certain contexts. Reliably achieving a lower $C_{\mathrm{ss}}$ may be preferred in patients for whom the target is moderate analgesia with clear avoidance of respiratory depressant effects. Alternatively, a higher $C_{\mathrm{ss}}$ may be preferred when using fentanyl to facilitate mechanical ventilation in a patient with severe lung disease and ventilator asynchrony, as the respiratory depressant effects may be desirable. More work is needed to clarify the magnitude of specific drug inducer effects in relation to fentanyl and to better understand the clinical implications of altered fentanyl hepatic metabolism as compared to hepatic extraction in the context of critical illness among children.

While greater knowledge of the pharmacokinetics and pharmacodynamics of the myriad drugs in critically ill children is needed, the dense sampling strategy required for traditional individual-level pharmacokinetic modeling is a significant barrier. Our approach using population pharmacokinetic modeling aligns with the strategic initiative outlined in the National Academy of Medicine's report on learning healthcare systems [39]. Pairing electronic data with remnant biologic samples provides an effective, minimally invasive, and cost-efficient approach to driving pharmacologic insights that would otherwise require substantial financial resources and potentially painful phlebotomy in a vulnerable population. Additional strengths of the current study include the relatively large cohort of children studied, the population pharmacokinetic model performance, and the introduction of relevant hepatic enzyme inducers as covariates.

While our cohort size constitutes the largest pharmacokinetic study of fentanyl in PICU patients, a notable limitation is that the present study is underpowered to identify associations with less common covariates, such as infrequently administered antibiotics that may interact with fentanyl and rare genotypes. Rifampin, for example, is a potent inducer of CYP3A4 but it is rarely administered and its effects on the hepatic metabolism of midazolam have been observed 2-4 weeks beyond discontinuation [40, 41]. Our model also did not account for de-induction and this should be a focus of future studies. Other potentially influential covariates not included in the present model include age-dependent CYP enzyme maturation, protein binding, BMI, enzyme and transporter function and related genotypes, hepatic disease, intra-abdominal pressure, and vasopressor use, to name a few. This is likely reflected by the high unaccounted for inter-individual variability in pharmacokinetic parameters. In addition, the unsuccessful inclusion of the CYP3A5 genotype in our model may be related to our relatively small cohort. In order to fully delineate the clinical significance of these covariates and better account for the complex heterogeneity of critically ill pediatric patients, additional pharmacokinetic studies of larger cohorts are necessary. 


\section{Conclusion}

We successfully constructed a population pharmacokinetic model for fentanyl using sparse samples collected at random times, demonstrating both the feasibility and utility of this approach for better understanding drug properties among one of the most heterogeneous patient populations in modern medicine. This work, which made use of existing electronic data and remnant samples at our quaternary children's hospital, demonstrates a feasible and effective learning health system approach to characterizing drug parameters among critically ill children. Larger population pharmacokinetic studies of fentanyl will be useful for honing our administration of this potent opioid, serving an eventual goal of developing a framework for precision administration of sedative and analgesic medications to critically ill children.

\section{Compliance with Ethical Standards}

Conflict of interest Fanuel T. Hagos, Christopher M. Horvat, Alicia K. Au, Yvette Conley, Lingjue Li, Samuel M. Poloyac, Patrick M. Kochanek, Robert S. B. Clark, and Philip E. Empey have no conflicts of interest relating to this article.

Funding This work was supported by NIH Grants 1TL1 TR00185801 (FTH) and NICHD T32 HD 040686 (CMH, PMK), the Children's Hospital of Pittsburgh Trust Young Investigator Award (CMH), and the Children's Hospital of Pittsburgh Scientific Fund (CMH). No sponsors were involved in the study design, collection, analysis, or interpretation of data, the writing of the report, or the decision to submit the manuscript for publication. Christopher M. Horvat wrote the first draft of this manuscript and no honorarium, grant, or other form of payment was given to produce this manuscript.

\section{References}

1. Curley MAQ, Wypij D, Watson RS, Grant MJC, Asaro LA, Cheifetz IM, et al. Protocolized sedation vs usual care in pediatric patients mechanically ventilated for acute respiratory failure: a randomized clinical trial. JAMA. 2015;313:379-89.

2. Sullivan KR, Cammarano WB, Wiener-Kronish JP. Analgesics, tranquilizers, and sedatives. Card intensive care. 2nd ed. Philadelphia: Elsevier/Saunders; 2010. p. 504-15. https://www.clini calkey.com/\#!/content/book/3-s2.0-B9781416037736100412 ?scrollTo=\%23hl0000403. Accessed 19 Mar 2018.

3. Ziesenitz VC, Vaughns JD, Koch G, Mikus G, van den Anker JN. Pharmacokinetics of fentanyl and its derivatives in children: a comprehensive review. Clin Pharmacokinet. 2018;57:125-49.

4. Van Driest SL, Marshall MD, Hachey B, Beck C, Crum K, Owen $\mathrm{J}$, et al. Pragmatic pharmacology: population pharmacokinetic analysis of fentanyl using remnant samples from children after cardiac surgery. Br J Clin Pharmacol. 2016;81:1165-74.

5. Peng PWH, Sandler AN. A review of the use of fentanyl analgesia in the management of acute pain in adults. Anesthesiol J Am Soc Anesthesiol. 1999;90:576-99.

6. Gourlay GK, Kowalski SR, Plummer JL, Cousins MJ, Armstrong PJ. Fentanyl blood concentration-analgesic response relationship in the treatment of postoperative pain. Anesth Analg. 1988;67:329-37.

7. Katz R, Kelly HW. Pharmacokinetics of continuous infusions of fentanyl in critically ill children. Crit Care Med. 1993;21:995-1000.

8. Frakes MA, Lord WR, Kociszewski C, Wedel SK. Efficacy of fentanyl analgesia for trauma in critical care transport. Am J Emerg Med. 2006;24:286-9.

9. Ziesenitz VC, König SK, Mahlke NS, Skopp G, Haefeli WE, Mikus G. Pharmacokinetic interaction of intravenous fentanyl with ketoconazole. J Clin Pharmacol. 2015;55:708-17.

10. Empey PE, Miller TM, Philbrick AH, Melick JA, Kochanek PM, Poloyac SM. Mild hypothermia decreases fentanyl and midazolam steady-state clearance in a rat model of cardiac arrest. Crit Care Med. 2012;40:1221-8.

11. Keizer RJ, van Benten M, Beijnen JH, Schellens JHM, Huitema ADR. Piraña and PCluster: a modeling environment and cluster infrastructure for NONMEM. Comput Methods Programs Biomed. 2011;101:72-9.

12. Lindbom L, Pihlgren P, Jonsson EN, Jonsson N. PsN-Toolkit-a collection of computer intensive statistical methods for non-linear mixed effect modeling using NONMEM. Comput Methods Programs Biomed. 2005;79:241-57.

13. Jonsson EN, Karlsson MO. Xpose-an S-PLUS based population pharmacokinetic/pharmacodynamic model building aid for NONMEM. Comput Methods Programs Biomed. 1999;58:51-64.

14. Choi L, Ferrell BA, Vasilevskis EE, Pandharipande PP, Heltsley R, Ely EW, et al. Population pharmacokinetics of fentanyl in the critically ill. Crit Care Med. 2016;44:64-72.

15. Horvat C, Au A, Conley Y, Kochanek P, Li L, Poloyac S, et al. ARDA2A and CYP3A5 genotypes are associated with fentanyl requirements in critically ill children [abstract no. 880]. Crit Care Med. 2018;46:424.

16. Singleton MA, Rosen JI, Fisher DM. Plasma concentrations of fentanyl in infants, children and adults. Can J Anaesth. 1987;34:152-5.

17. Encinas E, Calvo R, Lukas JC, Vozmediano V, Rodriguez M, Suarez E. A predictive pharmacokinetic/pharmacodynamic model of fentanyl for analgesia/sedation in neonates based on a semiphysiologic approach. Paediatr Drugs. 2013;15:247-57.

18. Koehntop DE, Rodman JH, Brundage DM, Hegland MG, Buckley JJ. Pharmacokinetics of fentanyl in neonates. Anesth Analg. 1986;65:227-32.

19. Anderson BJ, Allegaert K. The pharmacology of anaesthetics in the neonate. Best Pract Res Clin Anaesthesiol. 2010;24:419-31.

20. Ariano RE, Duke PC, Sitar DS. Population pharmacokinetics of fentanyl in healthy volunteers. J Clin Pharmacol. 2001;41:757-63.

21. Friis-Hansen B. Body composition during growth. In vivo measurements and biochemical data correlated to differential anatomical growth. Pediatrics. 1971;47(1 Suppl 2):264-72.

22. Weber DR, Leonard MB, Zemel BS. Body composition analysis in the pediatric population. Pediatr Endocrinol Rev PER. 2012;10:130-9.

23. Mulla H, Johnson TN. Dosing dilemmas in obese children. Arch Dis Child Educ Pract Ed. 2010;95:112-7.

24. Bower S, Hull CJ. Comparative pharmacokinetics of fentanyl and alfentanil. Br J Anaesth. 1982;54:871-7.

25. Ibrahim AE, Feldman J, Karim A, Kharasch ED. Simultaneous assessment of drug interactions with low- and high-extraction opioids: application to parecoxib effects on the pharmacokinetics and pharmacodynamics of fentanyl and alfentanil. Anesthesiology. 2003;98:853-61.

26. Kuip EJM, Zandvliet ML, Koolen SLW, Mathijssen RHJ, van der Rijt CCD. A review of factors explaining variability in fentanyl pharmacokinetics; focus on implications for cancer patients. Br J Clin Pharmacol. 2017;83:294-313. 
27. Feierman DE, Lasker JM. Metabolism of fentanyl, a synthetic opioid analgesic, by human liver microsomes. Role of CYP3A4. Drug Metab Dispos Biol Fate Chem. 1996;24:932-9.

28. Tateishi T, Krivoruk Y, Ueng YF, Wood AJ, Guengerich FP, Wood M. Identification of human liver cytochrome P-450 3A4 as the enzyme responsible for fentanyl and sufentanil N-dealkylation. Anesth Analg. 1996;82:167-72.

29. Takashina Y, Naito T, Mino Y, Yagi T, Ohnishi K, Kawakami J. Impact of CYP3A5 and ABCB1 gene polymorphisms on fentanyl pharmacokinetics and clinical responses in cancer patients undergoing conversion to a transdermal system. Drug Metab Pharmacokinet. 2012;27:414-21.

30. Zhou S-F. Drugs behave as substrates, inhibitors and inducers of human cytochrome P450 3A4. Curr Drug Metab. 2008;9:310-22.

31. Hukkanen J. Induction of cytochrome P450 enzymes: a view on human in vivo findings. Expert Rev Clin Pharmacol. 2012;5:569-85.

32. Burk O, Koch I, Raucy J, Hustert E, Eichelbaum M, Brockmöller $\mathrm{J}$, et al. The induction of cytochrome P450 3A5 (CYP3A5) in the human liver and intestine is mediated by the xenobiotic sensors pregnane $\mathrm{X}$ receptor (PXR) and constitutively activated receptor (CAR). J Biol Chem. 2004;279:38379-85.

33. Wortham M, Czerwinski M, He L, Parkinson A, Wan Y-JY. Expression of constitutive androstane receptor, hepatic nuclear factor 4 alpha, and P450 oxidoreductase genes determines interindividual variability in basal expression and activity of a broad scope of xenobiotic metabolism genes in the human liver. Drug Metab Dispos Biol Fate Chem. 2007;35:1700-10.
34. Landay RA, Gonzalez MA, Taylor JC. Effect of phenobarbital on theophylline disposition. J Allergy Clin Immunol. 1978;62:27-9.

35. Dahlqvist R, Steiner E, Koike Y, von Bahr C, Lind M, Billing B. Induction of theophylline metabolism by pentobarbital. Ther Drug Monit. 1989;11:408-10.

36. Wietholtz H, Zysset T, Kreiten K, Kohl D, Büchsel R, Matern S. Effect of phenytoin, carbamazepine, and valproic acid on caffeine metabolism. Eur J Clin Pharmacol. 1989;36:401-6.

37. Miller M, Cosgriff J, Kwong T, Morken DA. Influence of phenytoin on theophylline clearance. Clin Pharmacol Ther. 1984;35:666-9.

38. Ohnhaus EE, Breckenridge AM, Park BK. Urinary excretion of 6 beta-hydroxycortisol and the time course measurement of enzyme induction in man. Eur J Clin Pharmacol. 1989;36:39-46.

39. Olsen L, Aisner D, McGinnis JM, Institute of Medicine (U.S.), editors. The learning healthcare system: workshop summary. Washington, DC: National Academies Press; 2007.

40. Brodie MJ, Mintzer S, Pack AM, Gidal BE, Vecht CJ, Schmidt D. Enzyme induction with antiepileptic drugs: cause for concern? Epilepsia. 2013;54:11-27.

41. Reitman ML, Chu X, Cai X, Yabut J, Venkatasubramanian R, Zajic S, et al. Rifampin's acute inhibitory and chronic inductive drug interactions: experimental and model-based approaches to drug-drug interaction trial design. Clin Pharmacol Ther. 2011;89:234-42.

\section{Affiliations}

\section{Fanuel T. Hagos ${ }^{1}$. Christopher M. Horvat ${ }^{2,3}$ (1) - Alicia K. Au ${ }^{2,3}$ - Yvette P. Conley ${ }^{4,5}$ - Lingjue $\mathrm{Li}^{3,6}$. Samuel M. Poloyac ${ }^{3,6} \cdot$ Patrick M. Kochanek ${ }^{2,3} \cdot$ Robert S. B. Clark $^{2,3} \cdot$ Philip E. Empey ${ }^{3,6}$}

1 Center for Clinical Pharmaceutical Sciences, University of Pittsburgh School of Pharmacy, Pittsburgh, PA, USA

2 Department of Critical Care Medicine, University of Pittsburgh, School of Medicine and Children's Hospital of Pittsburgh of UPMC, 4401 Penn Ave, Faculty Pavilion Suite 2000, Pittsburgh, PA 1524, USA

3 Safar Center for Resuscitation Research, University of Pittsburgh, Pittsburgh, PA, USA
4 Department of Health Promotion and Development, University of Pittsburgh School of Nursing, Pittsburgh, PA, USA

5 Department of Human Genetics, University of Pittsburgh Graduate School of Public Health, Pittsburgh, PA, USA

6 Division of Pharmacy and Therapeutics, University of Pittsburgh School of Pharmacy, Pittsburgh, PA, USA 